\title{
Roles of Immunohistochemical Staining in Diagnosing Pulmonary Squamous Cell Carcinoma
}

\author{
Yue Yan $^{1 \&}$, Ya-Xiong Zhang ${ }^{1,2 \&}$, Wen-Feng Fang ${ }^{1 \&}$, Shi-Yang Kang ${ }^{1,2}$, Jian-Hua \\ Zhan $^{1}$, Nan Chen ${ }^{1}$, Shao-Dong Hong ${ }^{1}$, Wen-Hua Liang ${ }^{1}$, Yan-Na Tang ${ }^{1}$, Da-Cheng \\ $\mathrm{He}^{1,2}$, Xuan Wu${ }^{1}$, Li Zhang ${ }^{1 *}$
}

\begin{abstract}
Background: Differentiating morphologic features based on hematoxylin-eosin (HE) staining is the most common method to classify pathological subtypes of non-small-cell lung cancer (NSCLC). However, its accuracy and inter-observer reproducibility in pathological diagnosis of poorly differentiated NSCLC remained to be improved. Materials and Methods: We attempted to explore the role of immunohistochemistry (IHC) staining in diagnosing pulmonary squamous cell carcinoma (SQCC) with poorly differentiated features by HE staining or with elevated serum adenocarcinoma-specific tumor markers (AD-TMs). We also compared the difference of epidermal growth factor receptor (EGFR) mutation rate between patients with confirmed SQCC and those with revised pathological subtype. Logistic regression analyses were used to test the association between different factors and diagnostic accuracy. Results: A total of 132 patients who met the eligible criteria and had adequate specimens for IHC confirmation were included. Pathological revised cases in poor differentiated subgroup, biopsy samples and high-level AD-TMs cases were more than those with high/moderate differentiation, surgical specimens and normal-level AD-TMs. Moreover, biopsy sample was a significant factor decreasing diagnostic accuracy of pathological subtype (OR, 4.037; 95\% CI 1.446-11.267, $p=0.008)$. Additionally, EGFR mutation rate was higher in patients with pathological diagnostic changes than those with confirmed SQCC $(16.7 \%$ vs 4.4\%, $p=0.157$ ). Conclusions: Diagnosis based on HE staining only might cause pathological misinterpretation in NSCLC patients with poor differentiation or high-level AD-TMs, especially those with biopsy samples. HE staining and IHC should be combined as pathological diagnostic standard. The occurrence of EGFR mutations in pulmonary SQCC might be overestimated.
\end{abstract}

Keywords: Non-small-cell lung cancer - pulmonary squamous cell carcinoma - epidermal growth factor receptor

Asian Pac J Cancer Prev, 16 (2), 551-557

\section{Introduction}

Lung cancer is the leading cause of cancer death with the rapidest growth of morbidity and mortality all over the world during the past 50 years (Ferlay et al., 2007; Ferlay et al., 2010). In China, lung cancer also tops the list of mortality in all kinds of cancer among both male and female patients (Guo et al., 2012). The World Health Organization (WHO) divides lung cancer into two broad categories based on its biology, therapy, and prognosis: small-cell lung cancer (SCLC) and non-small cell lung cancer (NSCLC), which account for $15 \%$ 25\% and $70 \% \sim 85 \%$ of patients respectively (Beasley et al., 2005). Histologically, NSCLC includes adenocarcinoma (ADC), squamous cell carcinoma (SQCC), adenosquamous carcinoma (ADSQC), large-cell carcinoma (LCC) and other cell types. ADC and SQCC are major types of
NSCLC occurring in approximately $50 \%$ and $30 \%$ of cases (Ginsberg et al., 2007; Ettinger et al ., 2010).

Previously, the histologic subtyping of advanced NSCLC was not clinically or therapeutically important because of a lack of differential treatment options for NSCLC subtypes (Goldstraw et al., 1993; Thomas et al., 1993). However, the differential activity or limited indications of newer agents called for a distinction between SQCC and ADC of the lung. For instance, several clinical trials showed superior efficacy of pemetrexed in nonsquamous NSCLC, while SQCC obtained more benefit from gemcitabine (Scagliotti et al., 2008; Ciuleanu et al., 2009; Syrigos et al., 2010; Paz-Ares et al., 2012). The occurrence rate of epidermal growth factor receptor (EGFR) mutation was higher in ADC than SQCC (Park et al., 2009; Leighl, 2012) which caused ADC patients had better outcomes using EGFR tyrosine kinase inhibitors

${ }^{1}$ Sun Yat-sen University Cancer Center; State Key Laboratory of Oncology in South China; Collaborative Innovation Center for Cancer Medicine, ${ }^{2}$ Zhongshan School of Medicine, Sun Yat-sen University, Guangzhou, China \&Equal contributors *For correspondence: zhangli6@mail.sysu.edu.cn. 
(EGFR-TKIs) (Mok et al., 2009; Maemondo et al., 2010; Mitsudomi et al., 2010; Shukuya et al., 2011; Zhou et al., 2011; Han et al., 2012; Rosell et al., 2012). In addition, prior studies revealed increased risk of serious hemorrhage with bevacizumab in SQCC patients (Sandler et al., 2006; Cohen et al., 2007; Hapani et al., 2010). Therefore, it had highlighted the importance of specific pathological NSCLC subtyping before treatment.

Although the necessity of histologic subtyping had been increased, hematoxylin-eosin (HE) staining showed lower accuracy and interobserver reproducibility in diagnosis of poorly differentiated NSCLC, especially in small biopsy samples (Cataluna et al., 1996; Feinstein et al., 1970; Grilley-Olson et al., 2009; Travis et al., 2010; Sigel et al., 2011). Hence, many auxiliary methods, such as immunohistochemistry (IHC), gene expression profiling and micro RNA, had been studied to assist with this dilemma and IHC was the most convenient and practical solution (Johansson, 2004; Camilo et al., 2006; Kargi et al., 2007; Rossi et al., 2009). Several studies had shown that ADC and SQCC had virtually non-overlapping coexpression profiles of thyroid transcription factor 1 (TTF-1) and P63-the master transcriptional regulators in glandular and squamous cell lineages, respectively (Loo et al., 2010; Nicholson et al., 2010). Another research indicated that the best single marker for ADC and SQCC, based on sensitivity and specificity, would be Napsin A and P63, respectively (Noh et al., 2012). The latest studies showed an isoform of P63, P40, suggested to be equivalent to $\mathrm{P} 63$ in sensitivity for SQCC, but it is markedly superior to P63 in specificity (Bishop et al., 2012; Nobre et al., 2013). The combination of these IHC markers might be optimal.

Besides above IHC markers, there were many serum tumor markers (TMs) which indicated pulmonary ADC, such as carcinoembryonic antigen (CEA), cancer antigen 125 (CA125), CA153 and CA199 (Diez et al., 1991; Diez et al., 1994; Picardo et al., 1994; Mitsuhashi et al., 1995; Picardo et al., 1996; Alatas et al., 2001). Nevertheless, some SQCC cases diagnosed by HE staining showed elevated expression of these adenocarcinoma-specific tumor markers (AD-TMs). It was possible that a potential misinterpretation considering ADC with high-level ADTMs as SQCC based on HE staining might be existed.

In this study, we performed IHC testing using TTF-1, p63, Napsin A and p40 to verify the pathological type of pulmonary SQCC with poor differentiation or high-level AD-TMs diagnosed previously by HE staining. We also collected genetic data of these patients with known EGFR mutation status to compare the difference of EGFR gene mutations between patients with confirmed pathological diagnosis and those with revised pathological subtype.

\section{Materials and Methods}

\section{Patients}

2958 patients diagnosed with pulmonary SQCC who were treated at the Cancer Center of Sun Yat-Sen University (Guangzhou, China) during January 2007 to October 2012 were screened in the study. Only 132 patients with poor differentiation or high-level AD-TMs diagnosed previously by HE staining were enrolled onto the research ultimately if they had enough tissue sample. All of the records such as clinical and pathological features were collected with no patients having received any treatment. The clinicopathological features of the patients included age, gender, smoking history, UICC stage, degree of differentiation, level of serum AD-TMs, type of tissue sample and EGFR mutation status. All patients were restaged by seventh edition of UICC Staging System for NSCLC. Categories of each characteristic were divided as following: for age, patients more than 55 years old were considered as the older group. Smoking history was noted as yes or no. High AD-TMs level was defined, if serum CEA, CA125, CA153 or CA199 $\geq 5 \mathrm{ng} / \mathrm{ml}, 35 \mathrm{U} / \mathrm{ml}, 25 \mathrm{U} /$ $\mathrm{ml}, 35 \mathrm{U} / \mathrm{ml}$, respectively. Tissue samples were collected from surgery or biopsy. EGFR exon 19 deletions or exon 21 alterations were considered as EGFR mutants by fluorescent quatititive polymerase chain reaction (PCR). The study was approved by the Institutional Review Board of Sun Yat-Sen University Cancer Center (Guangzhou, China). All the patients had provided written informed consent before samples were collected.

\section{IHC analyses}

IHC staining was performed using mouse monoclonal anti-human antibodies for reconfirming the pathological diagnosis, including P63 (DAKO, 1:100), TTF-1 (DAKO, 1:100), P40 (DAKO, 1:100) and Napsin A (DAKO, 1:50). Sections with 5- $\mu$ m-thick were cut from the formalin-fixed, paraffin-embedded (FFPE) tumor block and then routinely deparaffinized and rehydrated. For antigen retrieval, slides were heated in a microwave oven for 30 minutes in citrate buffer solution $(\mathrm{pH}=7.4)$ and cooled slowly at room temperature for 20 minutes. After blocking the activity of endogenous peroxidase with $3 \%$ hydrogen peroxide for 8 minutes, the sections were treated with primary antibodies and incubated for 12 hours. Subsequently, the slides were rinsed in PBS three times and incubated in biotinylated secondary antibodies. After incubation, slides were washed again with PBS and then visualized using diaminobenzidine. Finally, Mayer's hematoxylin was used to counterstain the sections, which were then dehydrated and mounted.

P40 and P63 were the IHC markers which indicated SQCC, while Napsin A and TTF-1 supported ADC. As long as one of SQCC markers was positive and ADC markers were double-negative, the profile was considered as SQCC. Similarly, only if one of ADC markers was positive and SQCC markers were double-negative, the profile was further confirmed as ADC. If the markers of SQCC and ADC were co-expressed, the case was regarded as ADSQC. Besides, the quadruple-negative profile was diagnosed as NSCLC-not otherwise specified (NSCLCNOS). The reassessment process was shown in Figure 1.

Two pathologists who don't know the information of the patients were asked to independently assess the expression. Histologic $(\mathrm{H})$ scores were assigned by multiplying the percentage of stained cells $(0$, absent; $1,<25 \% ; 2,25 \%-50 \% ; 3,51 \%-75 \%$ and $4,>75 \%)$ by an intensity score ( 0 , absent; 1 , weak; 2 , moderate; and 3 , strong). After analyzing the distribution of $\mathrm{H}$ scores, 


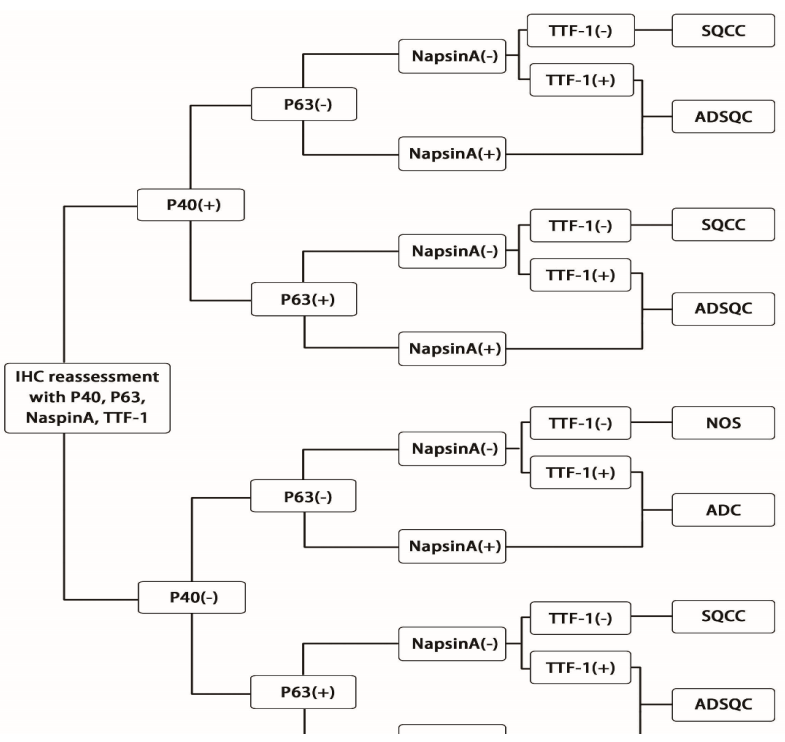

Figure 1. IHC Reassessment Chart for Pathological Diagnosis with P40, P63, Napsin A and TTF-1. Abbreviation: IHC=immunohistochemistry; TTF-1=thyroid transcription factor-1; SQCC=squamous cell carcinoma; $\mathrm{ADC}=$ adenocarcinoma; $\mathrm{ADSQC}=$ adenosquamous carcinoma; NOS $=$ not otherwise specified

Table 1. Baseline Data of Enrolled 132 Pulmonary SQCC Patients

\begin{tabular}{|c|c|c|}
\hline Characteristics & $\begin{array}{c}\text { Confirmed } \\
\text { pathological } \\
\text { diagnosis }\end{array}$ & $\begin{array}{c}\text { Revised } \\
\text { pathological } \\
\text { diagnosis }\end{array}$ \\
\hline \multicolumn{3}{|l|}{ Age [years] } \\
\hline$\leq 55$ & 36 & 6 \\
\hline$>55$ & 76 & 14 \\
\hline \multicolumn{3}{|l|}{ Gender } \\
\hline Male & 100 & 18 \\
\hline Female & 12 & 2 \\
\hline \multicolumn{3}{|l|}{ Smoking status } \\
\hline Yes & 94 & 15 \\
\hline No & 18 & 5 \\
\hline \multicolumn{3}{|l|}{ UICC stage } \\
\hline I-IIIA & 78 & 12 \\
\hline IIIB-IV & 34 & 8 \\
\hline \multicolumn{3}{|l|}{ Degree of differentiation } \\
\hline High \& moderate & 17 & 2 \\
\hline Low & 95 & 18 \\
\hline \multicolumn{3}{|l|}{ Level of AD-TMs } \\
\hline Normal & 63 & 8 \\
\hline High & 49 & 12 \\
\hline \multicolumn{3}{|l|}{ Type of tissue sample } \\
\hline Biopsy & 24 & 11 \\
\hline Surgery & 88 & 9 \\
\hline \multicolumn{3}{|l|}{ EGFR mutation status } \\
\hline Mutant & 4 & 3 \\
\hline Wild-type & 87 & 15 \\
\hline Unknown & 21 & 2 \\
\hline
\end{tabular}

*Abbreviations: AD-TMs=adenocarcinoma-specific tumor markers SQCC=squamous cell carcinoma

we set the results of IHC for positivity if the $\mathrm{H}$-score $\geq 4$ (Noh et al., 2012).

\section{Diagnostic standard of pathological type}

The combination of HE staining and IHC reassessment was the diagnostic standard of pathological subtype of
NSCLC. HE staining was the main methods to access the profile diagnosed as NSCLC-NOS. The final diagnosis will be discussed by two pathologists to reach consensus in case of disagreement.

\section{Statistical analysis}

SPSS 17.0 software was used for the statistical analysis. Continuous variables were divided into different categories as mentioned above. All the cut-off values were obtained by X-tile software (Version 3.6.1, Yale University, New Haven, CT), taking clinical expertise into consideration. The confirming rate of pathological diagnosis was the number of cases confirmed to be SQCC by the diagnostic standard divided by the number of cases diagnosed only by HE staining. Univariate and multivariate logistic regression analysis were used to test the association between the different factors and diagnostic accuracy. Results were reported with odds ratio (OR), corresponding $95 \%$ confidence intervals (CI). A two sided $p$-value $<0.05$ was considered statistically significant.

\section{Results}

\section{Patient characteristics}

A total of 132 patients with poor differentiation or high-level AD-TMs diagnosed as SQCC by HE staining previously were included in this study. The first cohort consisted of 61 patients with high-level AD-TMs who were divided into four subgroups according to the degree of differentiation and type of sample. The second cohort included 71 patients with normal-level AD-TMs and poor differentiation classified as two subgroups based on the type of sample (Figure 2). The median age of all the enrolled patients was 60 years (range: $30-90$ years). Most of the patients were male $(\mathrm{n}=118,89.4 \%)$ and smokers $(n=109,82.6 \%)$. More than half of the patients were in UICC III or IV stage $(n=78,59.1 \%)$. The majority of the tissue sample was from surgery $(n=97,73.5 \%)$. Most of the patients had known EGFR mutation status $(n=109$, $82.6 \%$ ). The basic clinical characteristics of the patients were presented in Table 1.

Pathological reassessment based on the combination of $H E$ staining and $I H C$ reassessment

132 patients in the two cohorts had adequate specimens for IHC reassessment. The pathological subtype diagnosed as SQCC was 112 cases (Figure 3A), while the revised diagnosis were 20 cases with $\mathrm{ADC}(\mathrm{n}=18)$ (Figure $3 \mathrm{~B})$, $\operatorname{ADSQC}(\mathrm{n}=1)$ (Figure 3C) and LCC $(\mathrm{n}=1)$ (not displayed) respectively. The pathological confirming rate of all cases was $84.8 \%$.

In the first cohort, the confirming rate in poor differentiated subgroup $(84.0 \%, 64.7 \%)$ was lower than high/moderate differentiated subgroup $(92.3 \%, 83.3 \%)$ whatever the specimen was collected from surgery or biopsy. In addition, biopsy samples had lower confirming rate $(83.3 \%, 64.7 \%)$ than surgical samples $(92.3 \%$, $84.0 \%$ ) both in high/moderate and poor differentiated subgroups, while the result was similar in the second cohort (confirming rate of biopsy samples and surgical samples $=66.7 \%$ and 93.2\%). Besides, patients with high- 
Table 2. Pathological Reassessment of 61 Pulmonary SQCC Patients with Gigh-level AD-TMs

\begin{tabular}{lcccc}
\hline Pathological reassessment & $\begin{array}{c}\text { Samples from surgery }(\mathrm{n}=38) \\
\text { High/moderate } \\
\text { differentiation } \\
(\mathrm{n}=13)\end{array}$ & $\begin{array}{c}\text { Poor } \\
\text { differentiation } \\
(\mathrm{n}=25)\end{array}$ & $\begin{array}{c}\text { Samples from biopsy }(\mathrm{n}=23) \\
\text { High/moderate } \\
\text { differentiation } \\
(\mathrm{n}=6)\end{array}$ & $\begin{array}{c}\text { Poor } \\
\text { differentiation } \\
(\mathrm{n}=17)\end{array}$ \\
\hline SQCC & 12 & 21 & 5 & 11 \\
ADC & 1 & 4 & 0 & 6 \\
ADSQC & 0 & 0 & 1 & 0 \\
Confirming rate & $92.30 \%$ & $84.00 \%$ & $83.30 \%$ & $64.70 \%$ \\
\hline
\end{tabular}

*Abbreviations: AD-TMs=adenocarcinoma-specific tumor markers; SQCC=squamous cell carcinoma; ADC=adenocarcinoma; ADSQC=adenosquamous carcinoma

Table 3. Pathological Reassessment of 71 Pulmonary SQCC Patients with Normal-level AD-TMs and Poor Differentiation

\begin{tabular}{lcc}
\hline $\begin{array}{l}\text { Pathological } \\
\text { reassessment }\end{array}$ & $\begin{array}{c}\text { Samples from surgery } \\
(\mathrm{n}=59)\end{array}$ & $\begin{array}{c}\text { Samples from biopsy } \\
(\mathrm{n}=12)\end{array}$ \\
\hline SQCC & 55 & 8 \\
ADC & 4 & 3 \\
LCC & 0 & 1 \\
Confirming rate & $93.20 \%$ & $66.70 \%$
\end{tabular}

*Abbreviations: AD-TMs=adenocarcinoma-specific tumor markers; $\mathrm{SQCC}=$ squamous cell carcinoma; $\mathrm{ADC}=$ adenocarcinoma; $\mathrm{LCC}=$ largecell carcinoma

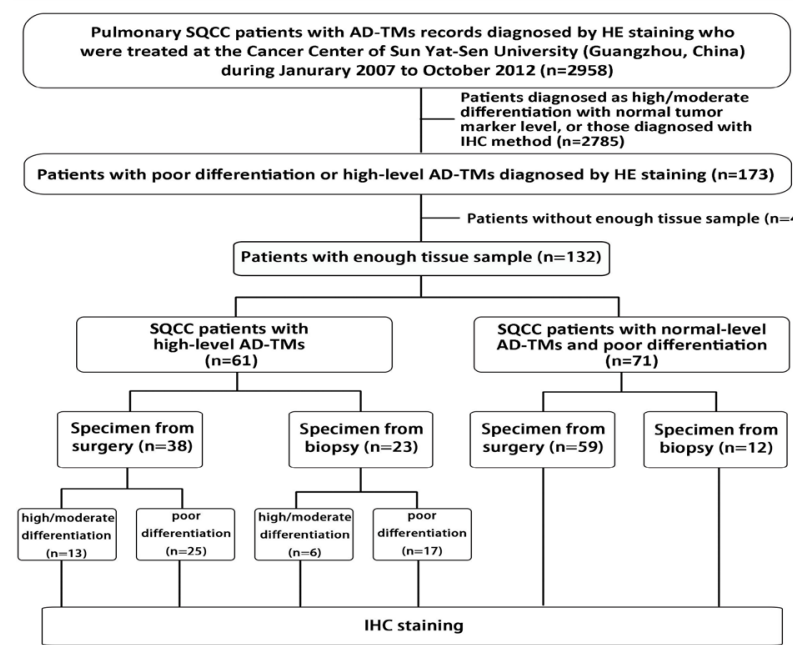

Figure 2. Flowing Chart of the Enrollment. Abbreviation: $\mathrm{AD}-\mathrm{TMs}=$ adenocarcinoma-specific tumor markers; $\mathrm{HE}=$ hematoxylin-eosin; $\mathrm{SQCC}=$ squamous cell carcinoma; $\mathrm{IHC}=$ immunohistochemistry

level AD-TMs had lower confirming rate $(84.0 \%, 64.7 \%)$ than those with normal-level AD-TMs $(93.2 \%, 66.7 \%)$ in poor differentiated cases whatever the specimen was from surgery or biopsy (Table 2, 3).

Univariate logistic regression analysis showed that biopsy sample was a significant factor which lowered diagnostic accuracy of pathological subtype (OR, 4.481; $p=0.002)$, while the poor differentiated sample (OR, 1.611; $p=0.737)$ or cases with high-level AD-TMs (OR, 1.929; $p=0.179$ ) were not. Additionally, multivariate logistic regression analysis testing the association between the different factors and pathological diagnostic accuracy showed the similar results (biopsy sample: OR, $4.03795 \%$ CI 1.446-11.267, $p=0.008$; poor differentiated sample: OR, $2.47895 \%$ CI $0.464-13.226, p=0.288$; cases with

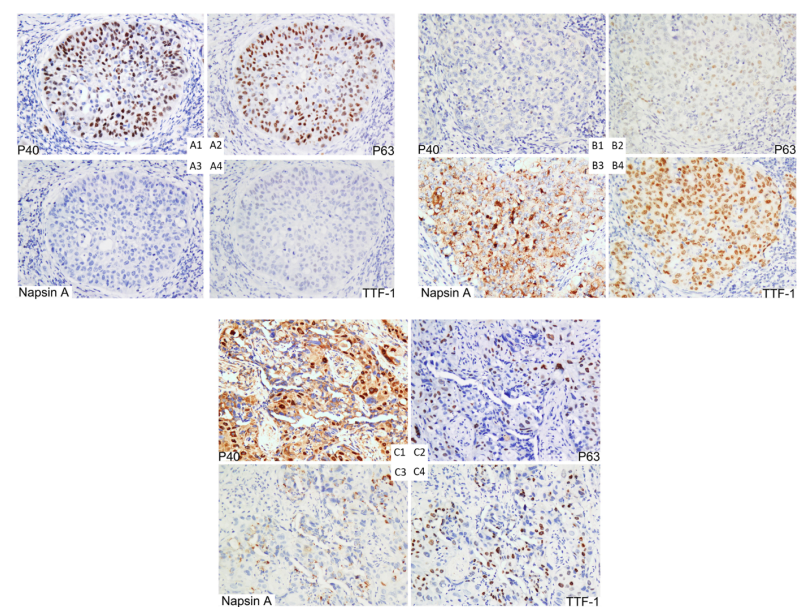

Figure 3. IHC Reassessment Of Pathological Diagnosis In Patients to be SQCC (A), ADC (B), ADSQC (C) (20x). (A1) P40 positive with deep-brown stained nuclei; (A2) P63 positive with brown stained nuclei; (A3) negative Napsin A without stained; (A4) negative TTF-1 sample without stained. (B1) P40 negative without stained; (B2) P63 negative without stained; (B3) positive Napsin A with deep-brown stained cytoplasm; (B4) positive TTF-1 sample with brown stained nuclei. (C1) positive P40 with brown stained nuclei; (C2) P63 positive with brown stained nuclei; (C3) positive Napsin A with slight-brown stained cytoplasm; (C4) positive TTF-1 sample with brown stained nuclei. Abbreviation: IHC=immunohistochemistry; TTF-1=thyroid transcription factor-1; SQCC=squamous cell carcinoma; $\mathrm{ADC}=$ adenocarcinoma; $\mathrm{ADSQC}=$ adenosquamous carcinoma

high-level AD-TMs: OR, 1.766 95\% CI 0.597-5.233, $p=0.304$ ) (Table 4).

\section{Pathological reassessment and EGFR mutation}

There were seven cases tested as EGFR mutants according to data of 109 patients with known EGFR mutation status through all the 132 included cases. Four EGFR mutants were in patients with revised pathological subtype and three mutants were in patients with confirmed pathological subtype. EGFR mutation rate was higher in patients with pathological diagnostic changes than those with confirmed pathological subtype after pathological reassessment $(16.7 \%, 3 / 18$ vs $4.4 \%$ 4/91, $p=0.157)$.

The pathological confirming rate in EGFR mutants was lower than EGFR wild-type patients $(57.1 \%, 4 / 7 \mathrm{vs}$ $85.3 \%$ 87/102). However, multivariate logistic regression analysis based on data of 109 patients with known EGFR mutation status showed that only biopsy sample was a significant factor which lowered diagnostic accuracy 
Table 4. The Association between the different Factors and Diagnostic Accuracy in Univariate and Multivariate Logistic Regression Analysis

\begin{tabular}{|c|c|c|c|c|c|c|c|}
\hline \multirow[t]{2}{*}{ Parameter } & \multirow{2}{*}{$\begin{array}{c}\text { Confirmed pathological } \\
\text { diagnosis }\end{array}$} & \multirow{2}{*}{$\begin{array}{c}\text { Revised pathological } \\
\text { diagnosis }\end{array}$} & \multicolumn{2}{|c|}{ Univariate analysis } & \multicolumn{3}{|c|}{ Multivariate analysis } \\
\hline & & & OR & $p$ value & OR & $95 \% \mathrm{CI}$ & $p$ value \\
\hline \multicolumn{8}{|c|}{ Degree of differentiation } \\
\hline Low & 95 & 18 & 1.611 & 0.737 & 2.478 & $0.464-13.226$ & 0.288 \\
\hline High \& moderate & 17 & 2 & 1 & & 1 & $(-)$ & \\
\hline \multicolumn{8}{|l|}{ Level of AD-TMs } \\
\hline High & 49 & 12 & 1.929 & 0.179 & 1.766 & $0.597-5.223$ & 0.304 \\
\hline Normal & 63 & 8 & 1 & & 1 & $(-)$ & \\
\hline \multicolumn{8}{|l|}{ Type of tissue sample } \\
\hline Biopsy & 24 & 11 & 4.481 & 0.002 & 4.037 & $1.446-11.267$ & 0.008 \\
\hline Surgery & 88 & 9 & 1 & & 1 & $(-)$ & \\
\hline
\end{tabular}

of pathological subtype (OR, $4.54095 \%$ CI 1.466$14.062, p=0.009)$ if taking EGFR mutation status into consideration.

\section{Discussion}

Evidence demonstrated that accurate pathological subtype classification was pivotal in selecting optimal chemotherapy (Scagliotti et al., 2008; Ciuleanu et al., 2009; Syrigos et al., 2010; Paz-Ares et al., 2012) and/or target therapy (Sandler et al., 2006; Cohen et al., 2007; Mok et al., 2009; Park et al., 2009; Hapani et al., 2010; Maemondo et al., 2010; Mitsudomi et al., 2010; Shukuya et al., 2011; Zhou et al., 2011; Han et al., 2012; Leighl, 2012; Rosell et al., 2012). Previous study reported that the agreement of pathological diagnosis of NSCLC based on HE-staining was correlated with differentiated degree of carcinoma, pathologist experience, pulmonary pathology expertise, pathologist diagnostic confidence and slide quality (Grilley-Olson et al., 2013). Another literature showed that $10 \%$ of SQCCs, $14 \%$ of ADCs and 50\% of LCCs were misclassified on bronchial biopsies (Cataluna et al., 1996). We also found some lung SQCC cases diagnosed by HE staining showed elevated expression of AD-TMs indicating ADC in clinical practice. Based on above all, we considered that some pulmonary SQCCs with poor differentiation, high-level AD-TMs or biopsy specimens diagnosed previously by HE staining might be doubtful in terms of pathological subtype.

Advances in IHC had brought great benefits to the field of pathological diagnosis of NSCLC. The latest research revealed that IHC testing was a significant factors associated with attaining a specific pathological diagnosis (Sulpher et al., 2013). However, too many articles tried to identify the most sensitive and specific IHC markers for pathological diagnosis (Loo et al., 2010; Nicholson et al., 2010; Noh et al., 2012; Bishop et al., 2012; Nobre et al., 2013) while few studies were focused on the practical effects reducing misinterpretation of pathological subtype by IHC tests in combination of the most important and reliable markers. Therefore, we use TTF-1, p63, Napsin A and p40 to verify the pathological type of pulmonary SQCC with poor differentiation or high-level AD-TMs diagnosed previously by HE staining.

Our results showed the occurrence of pathological subtype uncertainty in poor differentiated subgroup, biopsy samples and high-level AD-TMs cases was more than those with high/moderate differentiation, surgical specimens and normal-level AD-TMs. Moreover, biopsy sample was the significant factor decreasing diagnostic accuracy of pathological subtype. These results admit of following interpretations. Firstly, poor differentiated cell morphological features both in SQCC and ADC were immature and similar without obvious structures, such as intercellular bridge or acinus, to estimate, which might cause misinterpretation. Secondly, the specimen of biopsy was partial that could not represent general view of tumor. For example, if we only obtained limited tissue of ADSQC to test, either SQCC or ADC might be diagnosed. Other studies also reported that mixed cell types were seen in a small number of lung carcinoma cases (Roggli et al., 1985). Heterogeneity was identified by extensive sampling of the entire tumor and was seldom recognized in biopsy specimens (Roggli et al., 1985). Additionally, high-level AD-TMs were indicators of pulmonary ADC (Diez et al., 1991; Diez et al., 1994; Picardo et al., 1994; Mitsuhashi et al., 1995; Picardo et al., 1996; Alatas et al., 2001), which made the diagnosis of SQCC more questionable.

Another result in our study revealed that EGFR mutation rate was numerically higher in patients with pathological diagnostic changes than those with confirmed pathological subtype as SQCC after pathological reassessment. Previous studies reported that EGFR mutation rate was $12 \%-13.3 \%$ in Asian pulmonary SQCC (Tanaka et al., 2010; Hata et al., 2013), which was similar to the result of a meta-analysis that $10 \%$ of Chinese pulmonary SQCC had EGFR mutation (Wu et al., 2007). However, updated data of Catalogue of Somatic Mutations in Cancer (COSMIC) showed that EGFR mutation rate was only $5.1 \%$ in pulmonary SQCC (Forbes et al., 2011). Another research even suggested that EGFR mutation do not occur in pure pulmonary SQCC. Occasional detection of these mutations in samples diagnosed as SQCC is due to challenges with the diagnosis of ADSQC and ADC, which can be largely resolved by pathological reassessment by IHC biomarkers (Rekhtman et al., 2012). Based on previous results above and our result in terms of EGFR mutation rate of pulmonary SQCC (4.4\%), we considered that the occurrence of EGFR mutations in pulmonary SQCC might be overestimated because of confounding 
pathological subtype in ADC.

There are several major limitations of our study. First, this is a retrospective study. All the data were collected retrospectively. Second, small sample size might result in no statistically significant association between degree of differentiation/levels of AD-TMs and pathological diagnostic accuracy. Besides, EGFR mutation status was unknown in a small portion of included patients which might affect the result in the difference of EGFR gene mutations between patients with confirmed pathological diagnosis and those with revised pathological subtype. Nonetheless, regardless of above limitations, this study showed that HE staining was restricted in diagnosis of pathological subtype of NSCLC. Patients with high-level AD-TMs or diagnosed as poor differentiated pulmonary SQCC by HE staining might be recommended to have IHC testing to confirm pathological diagnosis, especially those with biopsy samples. Nevertheless, because all the IHC markers could not avoid false positive or negative diagnosis, the combination of HE staining and IHC should be diagnostic standard of pathological subtype of NSCLC.

In conclusion, it is vital to confirm pathological subtype of NSCLC which is associated with the treatment and survival of patients. HE staining might cause pathological doubtful interpretation in pulmonary SQCC patients with poor differentiation or high-level AD-TMs, especially those with biopsy samples, while IHC tests can enhance diagnostic accuracy of pathological subtype. In clinical practice, HE staining and IHC should be combined as diagnostic standard of pathological subtype of NSCLC.

\section{References}

Alatas F, Alatas O, Metintas M, et al (2001). Diagnostic value of CEA, CA15-3, CA 19-9, CYFRA 21- 1, NSE and TSA assay in pleural effusions. Lung Cancer, 31, 9-16.

Beasley MB, Brambilla E, Travis WD (2005). The 2004 World Health Organization classification of lung tumors. Semin Roentgenol, 40, 90-7.

Bishop JA, Teruya-Feldstein J, Westra WH, et al (2012). P40 $(\triangle \mathrm{Np} 63)$ is superior to $\mathrm{p} 63$ for the diagnosis of pulmonary squamous cell carcinoma. Modern Pathology, 25, 405-15.

Camilo R, Capelozzi VL, Siqueira SA, Del Carlo Bernardi F (2006). Expression of p63, keratin 5/6, keratin 7, and surfactant-A in non-small cell lung carcinomas. Hum Pathol, 37, 542-6.

Cataluna JJ, Perpina M, Greses JV, et al (1996). Cell type accuracy of bronchial biopsy specimens in primary lung cancer. Chest, 109, 1199-203.

Ciuleanu T, Brodowicz T, Zielinski C, et al (2009). Maintenance pemetrexed plus best supportive care versus placebo plus best supportive care for non-small-cell lung cancer: a randomised, double-blind, phase 3 study. Lancet, 374, 1432-40.

Cohen MH, Gootenberg J, Keegan P, Pazdur R (2007). FDA drug approval summary: bevacizumab (Avastin) plus Carboplatin and Paclitaxel as first-line treatment of advanced/metastatic recurrent nonsquamous non-small cell lung cancer. Oncologist, 12, 713-8.

Diez M, Cerdàn FJ, Ortega MD, et al (1991). Evaluation of serum CA 125 as a tumor marker in non-small cell lung cancer. Cancer, 67, 150-4.

Diez M, Torres A, Pollan M, et al (1994). Prognostic significance of serum CA125 assay inpatients with NSCLC. Cancer, 73, 1368-76.

Ettinger DS, Akerley W, Bepler G, et al (2010). Non-small cell lung cancer. J Natl Compr Canc Netw, 8, 740-801.

Feinstein R, Gelfman NA, Yesner R (1970). Observer variability in the histopathologic diagnosis of lung cancer. Am Rev Respir Dis, 101, 671-84.

Ferlay J, Autier P, Boniol M, et al (2007). Estimates of the cancer incidence and mortality in Europe in 2006. Ann Oncol, 18, 581-92.

Ferlay J, Shin HR, Bray F, et al (2010). Estimates of worldwide burden of cancer in 2008: GLOBOCAN 2008. Int J Cancer, 127, 2893-917.

Forbes SA, Bindal N, Bamford S, et al (2011). COSMIC: mining complete cancer genomes in the catalogue of somatic mutations in cancer. Nucleic Acids Res, 39, 945-50.

Ginsberg MS, Grewal RK, Heelan RT (2007). Lung cancer. Radiol Clin North Am, 45, 21-43.

Goldstraw P, Crowley J, Chansky K, et al (1993). The IASLC lung cancer staging project: proposals for the revision of the TNM stage groupings in the forthcoming (seventh) edition of the TNM Classification of malignant tumours $\mathbf{J}$ Thorac Oncol 2007; 2: 706-714. Thorax, 48, 1135-9.

Grilley-Olson JE, Hayes DN, Moore DT, et al (2009). Diagnostic reproducibility of squamous cell carcinoma (SC) in the era of histology-directed non-small cell lung cancer (NSCLC) chemotherapy: a large prospective study. J Clin Oncol, 27, 15

Grilley-Olson JE, Hayes DN, Moore DT, et al (2013). Validation of interobserver agreement in lung cancer assessment: hematoxylin-eosin diagnostic reproducibility for non-small cell lung cancer: the 2004 World Health Organization classification and therapeutically relevant subsets. Arch Pathol Lab Med, 137, 32-40.

Guo P, Huang ZL, Yu P, Li K (2012). Trends in cancer mortality in China: an update. Ann Oncol, 23, 2755-62.

Han JY, Park K, Kim SW, et al (2012). First-SIGNAL: first-line single-agent Iressa versus gemcitabine and cisplatin trial in never-smokers with adenocarcinoma of the lung. J Clin Oncol, 30, 1122-8.

Hapani S, Sher A, Chu D, Wu S (2010). Increased risk of serious hemorrhage with bevacizumab in cancer patients: a metaanalysis. Oncology, 79, 27-38.

Hata A, Katakami N, Yoshioka H, et al (2013). How sensitive are epidermal growth factor receptor-tyrosine kinase inhibitors for squamous cell carcinoma of the lung harboring EGFR gene-sensitive mutations? J Thorac Oncol, 8, 89-95.

Johansson L (2004). Histopathologic classification of lung cancer: Relevance of cytokeratin and TTF-1 immunophenotyping. Ann Diagn Pathol, 8, 259-67.

Kargi D, Gurel B, Tuna (2007). Gurel, B. Appl Histochem Mol Morph, 15, 415-20.

Leighl NB (2012). Treatment paradigms for patients with metastatic non-small-cell lung cancer: first-, second-, and third-line. Curr Oncol, 19, S52-8.

Loo PS, Thomas SC, Nicolson MC, Fyfe MN, Kerr KM (2010). Subtyping of undifferentiated non-small cell carcinomas in bronchial biopsy specimens. J Thorac Oncol, 5, 442-7.

Maemondo M, Inoue A, Kobayashi K, et al (2010). Gefitinib or chemotherapy for non-small-cell lung cancer with mutated EGFR. $N$ Engl J Med, 362, 2380-8.

Mitsudomi T, Morita S, Yatabe Y, et al (2010). Gefitinib versus cisplatin plus docetaxel in patients with non-small-cell lung cancer harbouring mutations of the epidermal growth factor receptor (WJTOG3405): an open label, randomised phase 3 trial. Lancet Oncol, 11, 121-8.

Mitsuhashi N, Takahashi T, Sakurai H, et al (1995). Establishment and characterization of a new human lung 
poorly differentiated adenocarcinoma cell line, GLL-11. Lung Cancer, 12, 13-24.

Mok TS, Wu YL, Thongprasert S, et al (2009). Gefitinib or carboplatin-paclitaxel in pulmonary adenocarcinoma. $N$ Engl J Med, 361, 947-57.

Nicholson AG, Gonzalez D, Shah P, et al (2010). Refining the diagnosis and EGFR status of non-small cell lung carcinoma in biopsy and cytologic material, using a panel of mucin staining, TTF-1, cytokeratin 5/6, and P63, and EGFR mutation analysis. $J$ Thorac Oncol, 5, 436-41.

Nobre AR, Albergaria A, Schmitt F (2013). P40: A p63 isoform useful for lung cancer diagnosis -a review of the physiological and pathological role of p63. Acta Cytol, 57, 1-8.

Noh S, Shim H (2012). Optimal combination of immunohistochemical markers for subclassification of non-small cell lung carcinomas: A tissue microarray study of poorly differentiated areas. Lung Cancer, 76, 51-5.

Park SH, Ha SY, Lee JI, et al (2009). Epidermal growth factor receptor mutations and the clinical outcome in male smokers with squamous cell carcinoma of lung. J Korean Med Sci, 24, 448-52.

Paz-Ares L, de Marinis F, Dediu M, et al (2012). Maintenance therapy with pemetrexed plus best supportive care versus placebo plus best supportive care after induction therapy with pemetrexed plus cisplatin for advanced non-squamous non-small-cell lung cancer (PARAMOUNT): a doubleblind, phase 3, randomised controlled trial. Lancet Oncol, 13, 247-55.

Picardo AL, Diez M, Torres A, et al (1996). Analysis of the prognostic significance of cytosolic determination of CA125, CEA, SCC in patients with NSCLC. Cancer, 77, 1066-72.

Picardo AL, Torres AJ, Maestro M, et al (1994). Quantitative analysis of carcinoembryonic antigen, squamous cell carcinoma antigen, CA 125, and CA 50 cytosolic content in non-small cell lung cancer. Cancer, 73, 2305-11.

Rekhtman N, Paik PK, Arcila ME, et al (2012). Clarifying the spectrum of driver oncogene mutations in biomarker-verified squamous carcinoma of lung: lack of EGFR/KRAS and presence of PIK3CA/AKT1 mutations. Clin Cancer Res, 18, 1167-76.

Roggli VL, Vollmer RT, Greenberg SD, et al (1985). Lung cancer heterogeneity: a blinded and randomized study of 100 consecutive cases. Hum Pathol, 16, 569-79.

Rosell R, Carcereny E, Gervais R, et al (2012). Erlotinib versus standard chemotherapy as first-line treatment for European patients with advanced EGFR mutation -positive non-smallcell lung cancer (EURTAC): a multicentre, open-label, randomised phase 3 trial. Lancet Oncol, 13, 239-46.

Rossi G, Pelosi G, Graziano P, Barbareschi M, Papotti M (2009). A reevaluation of the clinical significance of histological subtyping of non-small-cell lung carcinoma: diagnostic algorithms in the era of personalized treatments. Int J Surg Pathol, 17, 206-18.

Sandler A, Gray R, Perry MC, et al (2006). Paclitaxel-carboplatin alone or with bevacizumab for non-small-cell lung cancer. N Engl J Med, 355, 2542-50.

Scagliotti GV, Parikh P, von Pawel J, et al (2008). Phase III study comparing cisplatin plus gemcitabine with cisplatin plus pemetrexed in chemotherapy-naive patients with advancedstage non-small-cell lung cancer. J Clin Oncol, 26, 3543-51.

Shukuya T, Takahashi T, Kaira R, et al (2011). Efficacy of gefitinib for non-adenocarcinoma non-small-cell lung cancer patients harboring epidermal growth factor receptor mutations: a pooled analysis of published reports. Cancer Sci, 102, 1032-7.

Sigel CS, Moreira AL, Travis WD, et al (2011). Subtyping of non-small cell lung carcinoma: a comparison of small biopsy and cytology specimens. J Thorac Oncol, 6, 1849-56.

Sulpher JA, Owen SP, Hon H, et al (2013). Factors influencing a specific pathologic diagnosis of non-small-cell lung carcinoma. Clin Lung Cancer, 14, 238-44.

Syrigos KN, Vansteenkiste J, Parikh P, et al (2010). Prognostic and predictive factors in a randomized phase III trial comparing cisplatin-pemetrexed versus cisplatin-gemcitabine in advanced non-small-cell lung cancer. Ann Oncol, 21, 556-61.

Tanaka T, Matsuoka M, Sutani A, et al (2010). Frequency of and variables associated with the EGFR mutation and its subtypes. Int J Cancer, 126, 651-5.

Thomas JS, Lamb D, Ashcroft T, et al (1993). How reliable is the diagnosis of lung cancer using small biopsy specimens? report of a UKCCCR lung cancer working party. Thorax, 48, 1135-9.

Travis WD, Rekhtman N, Riley GJ, et al (2010). Pathologic diagnosis of advanced lung cancer based on small biopsies and cytology: a paradigm shift. J Thorac Oncol, 5, 411-4.

Wu YL, Zhong WZ, Li LY, et al (2007). Epidermal growth factor receptor mutations and their correlation with gefitinib therapy in patients with non-small cell lung cancer: a metaanalysis based on updated individual patient data from six medical centers in mainland China. J Thorac Oncol, 2, 430-9.

Zhou C, Wu YL, Chen G, et al (2011). Erlotinib versus chemotherapy as first-line treatment for patients with advanced EGFR mutation-positive non-small-cell lung cancer (OPTIMAL, CTONG-0802): a multicentre, openlabel, randomised, phase 3 study. Lancet Oncol, 12, 735-42. 